\title{
Quelques aspects de l'Endoparasitisme par les Acariens *
}

\author{
par A. FAIN
}

\section{Introduction}

Le parasitisme par les Acariens est très répandu chez les Vertébrés, spécialement les Oiseaux et les Mammifères, et il peut revêtir des formes très variées. On rencontre les Acariens non seulement à la surface du corps, mais également dans les cavités naturelles et même à l'intérieur des tissus.

Examinons rapidement leurs principales localisations parasitaires chez les Oiseaux et les Mammifères.

Une faune très nombreuse vit à la surface ou à l'intérieur des plumes chez les Oiseaux. Ce sont les «Acariens plumicoles» qui se nourrissent de la substance cornée de la plume ou des sécrétions élaborées au niveau du follicule plumeux.

Un autre groupe, tout aussi important en genres et espèces et peut-être encore plus spécialisé, est celui des «Acariens pilicoles» ou Listrophoridés. Ces Acariens vivent accrochés en permanence aux poils des Mammifères et ils se nourrissent également des déchets cornés comme les précédents.

Les follicules plumeux ou pileux sont occupés par un petit nombre de formes hautement spécialisées appartenant à des groupes très différents et nettement distincts des précédents.

La peau elle-même héberge une faune très riche et très disparate dans laquelle sont représentés les quatre grands sous-ordres d'Acariens. Certains de ces groupes comme les Tiques, les Mésostigmates et les Trombidiformes sucent le sang ou les liquides dermiques, alors que d'autres - et c'est le cas notamment des Sarcoptiformes - se nourrissent exclusivement aux dépens de la couche cornée de l'épiderme. Certains parmi ces derniers ne se contentent pas de brouter les couches superficielles de l'épiderme, mais pénètrent à l'intérieur de celles-ci en y creusant de longues galeries sinueuses. Cet envahissement de l'épiderme est observé principalement chez les Sarcoptidae, mais il existe aussi chez certains Trombidiformes comme les Psorergatidae, parasites de Mammifères, et les Ophioptidae vivant dans les écailles des Serpents. C'est parmi les Sarcoptiformes cuticoles et principalement ceux qui creusent des galeries cutanées que se recrutent la plupart des Acariens producteurs

* Travail présenté au Ier Congrès International de Parasitologie, Rome, 21-26 septembre 1964, et effectué à l'aide du Research Grant $\mathrm{n}^{\circ}$ 04870-02 du Public Health Service, Institute of Allergy and Infectious Diseases, Bethesda, Maryland, U.S.A. 
de gale. Les Sarcoptidae, ainsi que les quelques autres petits groupes qui ont élu domicile dans la peau, ne sont déjà plus de purs ectoparasites. Morphologiquement, ils montrent des signes évidents d'adaptation à la vie endoparasitaire qui les distinguent nettement des espèces vivant sur la peau. Personnellement, nous sommes enclins à les considérer plutôt comme des endoparasites.

A côté de cet endoparasitisme cutané, il existe aussi un endoparasitisme des muqueuses. Il n'a été rencontré que chez les Lémuriens et les Chauves-souris et seulement au niveau des muqueuses nasales et buccales. Les Acariens en cause sont enchâssés dans les couches superficielles de la muqueuse. Ils appartiennent aux sousordres des Sarcoptiformes et des Trombidiformes.

De toutes les formes d'acariase interne, la plus répandue est celle des voies respiratoires. On la rencontre principalement chez les Mammifères et les Oiseaux, mais elle existe aussi chez les Serpents, les Batraciens et même chez certains Invertébrés tels que les Mollusques pulmonés. Les Acariens sont présents à tous les niveaux de l'appareil respiratoire depuis l'œil jusqu'au poumon. Chez les Oiseaux, ils envahissent en outre les cellules osseuses du crâne et les sacs aériens. Tous ces Acariens des voies respiratoires sont spécifiques, non seulement en ce qui concerne l'hôte, mais encore pour la région souvent très limitée qu'ils occupent dans l'appareil respiratoire.

Une autre forme d'acariase interne est celle du tube digestif. Elle est beaucoup plus rare, et n'a été observée que chez les Mégachiroptères. Elle est produite par des petits Acariens de la famille Gastronyssidae qui vivent accrochés à la muqueuse de l'estomac et de l'intestin. La famille Gastronyssidae est encore représentée chez ces mêmes Chauves-souris par trois autres genres dont les représentants vivent, soit dans les fosses nasales, soit accrochés à la cornée oculaire. Il ne fait donc aucun doute que l'espèce qui vit dans l'estomac des Roussettes est un parasite authentique et non un faux parasite introduit avec la nourriture.

Nous terminerons cet aperçu général sur le parasitisme par les Acariens en rappelant la curieuse biologie des Hypopes. Les Hypopes sont des nymphes d'un type particulier produites par certains Sarcoptiformes, et notamment divers plumicoles, lorsque les conditions extérieures deviennent défavorables, par exemple à la suite d'un abaissement de la température extérieure ou encore au moment de la mue de l'Oiseau. Les Hypopes sont des formes de résistance dépourvues de bouche et d'appendices buccaux et donc incapables de se nourrir. Ces nymphes vont chercher refuge dans la profondeur du corps. Elles s'arrêtent habituellement sous la peau, à la surface des muscles; mais quelquefois elles envahissent aussi les tissus profonds allant même jusqu'à tapisser la surface externe de certains viscères comme les poumons et même le cœur.

Cet aperçu très général sur les principales formes du parasitisme chez les Acariens n'a d'autre but que de souligner le grand pouvoir d'adaptation de ces parasites à des conditions de vie très variées. Aucun autre groupe d'Arthropodes parasites ne présente une diversité aussi grande d'habitats. Ces remarquables aptitudes au parasitisme font des Acariens des témoins particulièrement sensibles dans l'étude des grands phénomènes de la vie parasitaire. 
Ajoutons encore que les Acariens constituent un des rares groupes de parasites qui renferment à la fois des formes libres, des ectoparasites et des endoparasites. Les chances de pouvoir retrouver des lignées phylogéniques complètes et progressives sont donc très grandes. Des conditions aussi favorables ne se retrouvent chez aucun autre groupe de parasites. Chez la plupart des Vers, les formes ectoparasites ont disparu ou n'ont jamais existé. Chez les Insectes, c'est au contraire l'ectoparasitisme qui est pratiquement la seule forme de parasitisme rencontrée.

Toutes ces considérations montrent que les Acariens sont des parasites qui se prêtent particulièrement bien à l'étude des phénomènes parasitaires en général et de l'endoparasitisme en particulier.

\section{Acariase respiratoire chez les Oiseaux}

Après ces quelques données générales sur le parasitisme par les Acariens, examinons maintenant plus en détail certains aspects intéressants de l'acariase respiratoire.

Nous avons dit que cette forme de parasitisme était très répandue chez les Oiseaux et les Mammifères et qu'elle existait également chez les Serpents, les Batraciens et les Mollusques pulmonés.

Chez les Oiseaux, les Acariens parasites des voies respiratoires appartiennent à quatre familles différentes : ce sont les Rhinonyssidae qui font partie du sous-ordre des Mesostigmata, les Ereynetidae du sous-ordre des Trombidiformes, les Cytoditidae et les Turbinoptidae appartenant tous deux au sous-ordre des Sarcoptiformes. Ces quatro familles groupent ensemble près de 300 espèces rangées dans 31 genres. Tous ces Acariens ne sont pas mélangés au hasard dans les voies respiratoires, mais ils occupent des places différentes en rapport essentiellement avec leur mode d'alimentation. Les Turbinoptidae, proches parents et dérivant probablement des Psoroptidae, sont les plus superficiels. Ils vivent dans les narines et se nourrissent, pensons-nous, comme tous les Acaridiae ectoparasites, de la substance cornée de la peau. Les Acariens de la deuxième famille, celle des Rhinonyssidae sont hématophages comme la plupart des Mésostigmates ectoparasites dont ils dérivent probablement. On les rencontre également dans les régions antérieures des fosses nasales, mais uniquement sur les parties les plus vascularisées des cornets. Une espèce cependant (Sternostoma tracheacolum) a envahi les bronches et les poumons où elle se nourrit d'ailleurs également de sang. Les Acariens formant la troisième famille, celle des Ereynetidae occupent les parties profondes des fosses nasales. Ils semblent se nourrir exclusivement du mucus comme le font probablement aussi leurs proches parents vivant dans les voies respiratoires des Mollusques, des Batraciens et des Mammifères. Enfin, les Acariens de la famille Cytoditidae ne comprennent que deux genres, l'un Cytonyssus vit dans les fosses nasales et les cavités osseuses de la tête, l'autre Cytodites n'est rencontré que dans les poumons et les sacs aériens. Ces Acariens semblent se nourrir des sérosités ou de la graisse qui transsudent à travers la muqueuse. 
La présence de ces Acariens dans les voies respiratoires soulève divers problèmes d'un grand intérêt qui touchent à l'essence même du parasitisme.

L'un des plus importants est celui de savoir comment le parasitisme à pris naissance. En d'autres termes, ces endoparasites proviennent-ils des ectoparasites vivant sur le corps ou dérivent-ils directement des formes libres ? On peut aussi se demander de quelle façon la biologie des hôtes a contribué à propager le parasitisme d'un groupe à l'autre. Voyons dans quelle mesure nous pouvons répondre à ces deux questions. Pour ne pas trop allonger cet exposé nous nous limiterons aux deux familles les mieux connues : Ereynetidae et Rhinonyssidae.

\section{Etudions d'abord la famille Ereynetidae.}

Cette famille comprend environ 75 espèces. Dans ce nombre, il y a une douzaine d'espèces libres, une espèce qui vit dans le poumon des Mollusques pulmonés, et 62 espèces parasites des fosses nasales de Vertébrés. Parmi celles-ci, 9 vivent chez les Batraciens, $43 \mathrm{chez}$ les Oiseaux et $10 \mathrm{chez}$ les Mammifères. Fait remarquable, il n'existe dans ce groupe aucune espèce ectoparasite. D'autre part, on ne trouve sur le corps des Vertébrés aucune espèce de Trombidiformes qui pourrait présenter une affinité quelconque avec les Ereynetidae. Il semble donc bien que les espèces nasicoles des Vertébrés dérivent directement des formes libres. L'adaptation au parasitisme est probablement déjà très ancienne, car les espèces endonasales sont morphologiquement beaucoup plus évoluées que les espèces libres et elles appartiennent à des sous-familles différentes. A cette règle il y a cependant une exception. C'est celle de Ereynetoides malayi, une espèce provenant des fosses nasales d'un petit nectarin du genre Arachnothera. Elle appartient à un genre très primitif qui en dehors de cette seule espèce ne comporte que des formes libres. Le parasitisme de cet Oiseau est cependant indiscutable, car il a été observé à plusieurs reprises et à différents moments de l'année. De plus, les Acariens étaient représentés non seulement par les formes adultes, mais également par toute la série des stades immatures. Il semble donc bien que l'on soit en présence d'un cas de parasitisme véritable, mais très récent (6).

Cet exemple prouve que les Ereynetidae libres sont capables de passer d'emblée à la vie endoparasitaire sans passer par le stade d'ectoparasite.

C'est vraisemblablement de cette façon que les choses se sont passées pour toutes les autres espèces parasites de ce groupe. L'invasion des fosses nasales était probablement, à l'origine, un simple accident. Si l'Acarien a pu se maintenir dans ce nouvel habitat et même s'y reproduire, c'est qu'il y a trouvé un minimum de conditions favorables. La biologie de ces Acariens est encore très mal connue, mais l'observation a montré que toutes les formes libres vivent dans des milieux à très forte humidité relative. Or on sait que les Acariens sont très sensibles à des variations même minimes dans ce domaine. Le succès des Ereynetidae dans leur envahissement des voies respiratoires pourrait s'expliquer par le fait qu'ils ont trouvé dans ce biotope des conditions d'humidité semblables à celles qui régnaient dans le milieu naturel. 
Une deuxième condition d'adaptation est la nourriture. On ne connaît encore rien de précis sur les besoins des Ereynetidae dans ce domaine. Il semble que certaines espèces libres se nourrissent d'algues ou de champignons microscopiques. Quant aux espèces parasites, on sait seulement qu'elles n'absorbent pas de sang et qu'elles vivent à la surface de muqueuses sécrétant du mucus. Il est donc probable qu'elles se nourrissent toutes de mucus.

Un autre point intéressant à envisager est la spécificité. Après ce que nous venons de dire, on serait porté à croire que la spécificité est très peu marquée chez ces Acariens. En fait elle est moins stricte que chez les autres Acariens endoparasites, mais elle est néanmoins évidente.

La spécificité est très peu marquée à l'échelon générique. Tous les genres sont représentés chez au moins deux ordres d'Oiseaux. Le genre Neoboydaia par exemple est rencontré chez sept ordres différents. Notons toutefois que toutes les espèces qui vivent chez les Passériformes, soit 21 au total, appartiennent à un seul genre (Boydaia); mais celui-ci renferme encore deux espèces parasites de Psittaciformes, deux autres parasites de Galliformes et une vivant chez les Falconiformes.

La spécificité est relativement bien marquée à l'échelon de l'espèce. La présence d'une espèce chez deux ordres d'Oiseaux est exceptionnelle. Elle n'a été observée que deux fois et dans l'un de ces cas il s'agissait très probablement d'une infestation accidentelle.

Généralement on retrouve la même espèce chez plusieurs genres, plus rarement chez tous les genres, d'une même famille d'hôtes. Trois espèces seulement sur un total de 42 sont rencontrées régulièrement chez deux ou trois familles d'hôtes. Ce cas se présente notamment pour Boydaia nigra. Cette espèce est régulièrement rencontrée au Ruanda chez le moineau domestique, un Ploceidae, ainsi que chez divers serins et hochequeues respectivement des Fringillidae et des Motacillidae. Tous ces petits Oiseaux font partie de ce que l'on pourrait appeler la faune subdomestique qui est très fréquente en Afrique Centrale. Ces Oiseaux sont attirés par les déchets alimentaires de toutes sortes qui traînent toujours autour des habitations. Ils arrivent ainsi en contact l'un avec l'autre et dès lors peuvent échanger leurs parasites. Il est vraisemblable que c'est de cette façon que Boydaia nigra a pu passer des Fringillidae qui semblent être les hôtes primitifs, aux autres familles de Passériformes.

La deuxième espèce qui fait exception à la règle de la spécificité est Boydaia spatulata. Cette espèce parasite sept genres de Turdidae, trois genres de Sylviidae et un Paridae: Parus niger. Le fait de la rencontrer chez les Turdidae et les Sylviidae ne doit pas nous étonner, car ces deux familles sont voisines. Sa présence chez un Paridae est moins explicable. En fait elle n'a été signalée qu'une seule fois chez cet hôte, et peut-être s'agissait-il d'un parasitisme accidentel.

La troisième espèce à faire exception au point de vue spécificité est Speleognathopsis galli, très fréquente chez la poule qui est un Phasianidae, et qui a été rencontrée une fois chez une pintade qui est un Numididae. 
Après cet aperçu sur la spécificité des Ereynetidae et sur les conditions écologiques qui ont pu favoriser leur dissémination, voyons maintenant quelle a été l'influence de la vie parasitaire sur le développement postembryonnaire chez ces Acariens (2 et 4).

Tous les Ereynetidae libres, ainsi que l'unique espèce qui vit chez les Mollusques sont ovipares. A l'œuf succède la larve, laquelle est elle-même suivie de trois types différents de nymphes qui sont, par ordre chronologique la protonymphe, la deutonymphe et la tritonymphe. Cette dernière donne naissance à l'adulte.

Chez les espèces qui vivent dans les fosses nasales des Batraciens, le développement est accéléré. La femelle pond des œufs qui renferment une larve déjà complètement développée. En outre il n'y a plus que deux stades nymphaux, le stade tritonymphe ayant disparu.

L'évolution est encore beaucoup plus écourtée chez les espèces qui parasitent les animaux à sang chaud, Oiseaux et Mammifères. Ces espèces sont également ovovivipares. La larve existe, mais il n'y a plus de stade nymphal et l'adulte se développe directement à l'intérieur de la dépouille larvaire (4).

La tendance à la viviparité et à l'accélération du développement postembryonnaire sous l'influence de la vie parasitaire est un phénomène général chez les Acariens parasites. Elle est d'autant plus marquée que l'Acarien est plus étroitement adapté à la vie endoparasitaire.

Parallèlement à ces profondes modifications dans le développement postembryonnaire, l'endoparasitisme a aussi provoqué chez les Ereynetidae des transformations morphologiques très importantes. Le temps nous manque pour les étudier en détail. Disons seulement que l'adaptation au parasitisme a provoqué chez toutes les espèces un retour à l'état larvaire. Le nombre des poils est en effet toujours plus réduit chez les espèces parasites que chez les formes libres, alors que chez les larves de ces groupes la chaetotaxie est presque identique (4).

C'est par l'étude de la chaetotaxie qu'on peut le mieux apprécier le degré de néoténie d'une espèce. La néoténie peut varier notablement au sein d'un même genre. Elle est parfois très marquée au point que chez certaines espèces l'adulte présente presque la même chaetotaxie que la larve correspondante.

Signalons enfin que l'adaptation à la vie parasitaire a encore provoqué chez les Ereynetidae une modification dans le dimorphisme sexuel. Chez les espèces libres, le mâle se distingue facilement de la femelle grâce à la forme différente de la fente sexuelle et à la présence d'un pénis. Chez les formes parasites, ces caractères sexuels primaires sont peu distincts et ils peuvent même s'effacer complètement chez certaines espèces très évoluées. En revanche chez ces dernières, on peut voir apparaître des nouveaux caractères situés en dehors de la zone sexuelle et qui sont des véritables caractères sexuels secondaires. Ils consistent habituellement dans le renflement considérable des poils au niveau de certaines pattes. Ce renflement qui peut parfois atteindre des proportions monstrueuses n'est observé que chez la femelle (3). 
Etudions maintenant la deuxième famille d'Acariens nasicoles, celle des Rhinonyssidae.

Ces Acariens sont plus répandus que les précédents. Le nombre d'espèces décrites jusqu'ici dans cette famille s'élève à plus de 200. Cet inventaire est toutefois encore très incomplet du fait que de nombreuses familles d'Oiseaux n'ont pas encore été explorées.

Ce groupe présente des différences notables avec le précédent. En effet toutes les espèces connues sont strictement inféodées aux voies respiratoires des Oiseaux, et la plupart d'entre elles se nourrissent exclusivement de sang.

Les ancêtres des Rhinonyssidae doivent être recherchés parmi les groupes de Mésostigmates vivant en ectoparasites sur les Oiseaux et tout spécialement les Dermanyssidae. Ils sont toutefois beaucoup plus évolués que ces derniers et présentent notamment une réduction notable de la chaetotaxie et du degré de chitinisation du corps. En outre le stigmate respiratoire s'est déplacé sur la face dorsale du corps, ce qui semble être une adaptation destinée à soustraire les orifices respiratoires au mucus nasal dans lequel ces Acariens sont englués.

La spécificité des Rhinonyssidae varie selon qu'on envisage le genre ou l'espèce. Certains genres sont presque complètement inféodés à des groupes écologiques d'hôtes. Ainsi par exemple, le genre Rhinonyssus comprend douze espèces toutes parasites d'Oiseaux aquatiques. Ces hôtes se répartissent comme suit : les Charadriiformes en hébergent sept espèces; les Podicipédiformes trois espèces; les Sphénisciformes et les Ansériformes chacun une espèce *.

La présence d'un même genre de parasites chez des hôtes aussi différents du point de vue zoologique peut paraître assez étonnante. Peut-être faut-il en chercher l'explication dans le fait que l'habitat aquatique favorise l'échange des parasites, ce qui pourrait freiner la spéciation. A l'appui de cette hypothèse, nous voyons en effet que les espèces du genre Rhinonyssus se différencient l'une de l'autre par des caractères qui sont habituellement moins tranchés que dans les autres genres. Nous constatons que c'est chez les Oiseaux à habitudes grégaires que le parasitisme est le plus fréquent mais aussi le plus uniforme. Les Anatidae par exemple ne sont parasités que par une seule espèce (Rhinonyssus rhinoletrum), mais celle-ci est rencontrée chez plus de quarante espèces d'Anatidae représentant vingt genres différents ( 1 et 7 ).

Les deux espèces connues du genre Larinyssus parasitent les Charadriiformes. L'une vit chez les Laridae, l'autre chez les Glareolidae. Remarquons aussi que les deux genres Rallinyssus et Rallinyssoides, comptant ensemble six espèces, sont endémiques pour les Rallidae (Gruiformes). Ces deux genres sont morphologiquement plus proches du genre Rhinonyssus que de la plupart des autres genres connus dans la famille.

Voyons maintenant quels sont les genres qui vivent exclusivement ou en ordre principal chez des hôtes non aquatiques.

* La classification des oiseaux adoptée ici est celle de A. Wetmore (A Classification for the Birds of the World, Smithsonian Inst., June 1960). 
Les genres Passeronyssus (neuf espèces) et Ruandanyssus (une espèce) sont complètement inféodés aux Passériformes.

Le genre Ptilonyssus comprend plus de quatre-vingts espèces parasites de Passériformes et huit espèces vivant chez divers autres ordres d'Oiseaux : Falconiformes (trois espèces), Coraciiformes (deux espèces), Apodiformes (deux espèces) et Piciformes (une espèce).

Le genre Sternostoma est également très répandu chez les Passériformes (27 espèces), mais on le rencontre aussi chez neuf autres ordres, mais seulement par une espèce dans chaque ordre: Strigiformes, Gruiformes, Charadriiformes, Galliformes, Coraciiformes, Cuculiformes, Coliiformes, Apodiformes, Piciformes.

Le genre Mesonyssoides est représenté par cinq espèces chez les Psittaciformes, une espèce chez les Piciformes et une espèce chez les Passériformes.

Le genre Astridiella comprend deux espèces parasites de Caprimulgiformes, deux parasites de Gruiformes (Otididae) et une vivant chez des Passériformes.

Le genre Tinaminyssus est formé de quatre espèces parasitant les Tinamiformes et une les Passériformes.

En dépit de quelques exceptions, on peut dire que les Passériformes hébergent une faune caractéristique, qui diffère nettement de celles des autres groupes d'Oiseaux. Notons encore que les espèces qui parasitent les Passériformes sont en général inféodées à une famille déterminée d'hôtes, cependant dans plusieurs familles d'Oiseaux, la spécificité semble liée à un groupe de genres ayant rang de sous-famille. Ce cas se présente notamment pour les Ploceidae dont les quatre sous-familles Ploceinae, Passerinae, Estrildinae et Viduinae sont parasitées par des espèces qui semblent leur être inféodées.

Signalons que les Rhinonyssidae comportent encore six autres genres, en dehors de ceux que nous venons de citer. Parmi ceux-ci, Rhinoecius (six espèces) et Zumptnyssus (une espèce) sont inféodés aux Strigiformes. Le genre Psittanyssus (une espèce) est endémique pour les Psittaciformes. Le genre Rhinosterna comprend une espèce parasite des Psittaciformes et une autre vivant chez les Apodiformes. Enfin le genre Mesonyssus est représenté par dix-neuf espèces réparties dans sept ordres d'Oiseaux. Neuf espèces vivent chez les Columbiformes, trois chez les Ciconiiformes, deux chez les Falconiformes, deux chez les Coraciiformes, une chez les Tinamiformes, une chez les Galliformes et une chez les Apodiformes.

Au lieu d'envisager la distribution des genres de Rhinonyssidae parmi les divers ordres d'Oiseaux, on peut aussi considérer la faune globale qui parasite chaque ordre en particulier. Nous voyons ainsi qu'il n'existe aucun ordre d'Oiseaux dont la faune soit complètement endémique à l'échelon générique. Chez tous les ordres d'Oiseaux, on rencontre au moins un genre de Rhinonyssidae qui est représenté également chez un ou plusieurs autres ordres d'Oiseaux.

Les Sphénisciformes, les Podicipédiformes et les Ansériformes sont parasités seulement par le genre Rhinonyssus, respectivement par une, trois et une espèces.

Les Tinamiformes sont parasités par deux genres ubiquistes: Tinaminyssus (trois espèces) et Mesonyssus (une espèce). 
Les Ciconiiformes (Ardeidae) et les Columbiformes hébergent seulement le genre ubiquiste Mesonyssus (respectivement 3 et 9 espèces).

Les Galliformes hébergent deux genres ubiquistes: Sternostoma et Mesonyssus, représentés chacun par une espèce.

Chez les Gruiformes, nous trouvons d'une part les Rallidae qui hébergent trois genres dont deux endémiques (Rallinyssus et Rallinyssoides, avec six espèces au total) et un ubiquiste (Sternostoma, avec une espèce), et d'autre part les Otididae, parasités seulement par le genre ubiquiste Astridiella (avec deux espèces).

Les Charadriiformes sont parasités par un genre endémique Larinyssus (une espèce chez les Laridae et une espèce chez les Glareolidae) et par deux genres ubiquistes: Rhinonyssus (sept espèces chez les Charadriidae, les Scolopacidae et les Alcidae) et Sternostoma (une seule espèce parasitant les Charadriidae, les Laridae et les Phalaropodidae).

Chez les Psittaciformes, le genre dominant est Mesonyssoides (cinq espèces) qui est représenté également chez les Piciformes et les Passériformes. On y rencontre en outre deux autres genres: Rhinosterna (une espèce) qui existe aussi chez les Apodiformes et Psittanyssus (une espèce) qui est endémique. Signalons encore que Sternostoma tracheacolum qui parasite de nombreux Passériformes, a également été rencontré chez des perruches d'élevage.

Les deux ordres des Cuculiformes et des Coliiformes hébergent chacun une espèce du genre Sternostoma.

Les Strigiformes hébergent trois genres de Rhinonyssidae dont deux sont endémiques (Rhinoecius et Zumptnyssus groupant ensemble 7 espèces) et un est ubiquiste (Sternostoma, avec une espèce).

Les Caprimulgiformes n'hébergent que le genre ubiquiste Astridiella (deux espèces).

Chez les Apodiformes, les Apodidae sont parasités par une espèce du genre Rhinosterna et par une espèce du genre Ptilonyssus, alors que les Trochilidae hébergent trois espèces appartenant à trois genres ubiquistes : Ptilonyssus, Sternostoma et Mesonyssus.

Les Coraciiformes sont parasités par trois genres ubiquistes: Mesonyssus (deux espèces), Ptilonyssus (deux espèces) et Sternostoma (une espèce).

Les Piciformes sont parasités par trois genres ubiquistes: Mesonyssoides (une espèce), Ptilonyssus (une espèce) et Sternostoma (une espèce).

Les Passériformes hébergent deux genres endémiques: Passeronyssus (neuf espèces) et Ruandanyssus (une espèce) et cinq genres ubiquistes. Parmi ces derniers, les genres Sternostoma et Ptilonyssus sont représentés respectivement par 27 et 85 espèces. Les trois autres genres sont Mesonyssoides (une espèce), Tinaminyssus (une espèce) et Astridiella (une espèce).

L'importance que peuvent présenter les facteurs écologiques dans la dissémination des Acariens de la famille Rhinonyssidae est bien mise en évidence pour Sternostoma tracheacolum. Cette espèce est la seule de tout ce groupe à parasiter les 
voies respiratoires inférieures : trachée, poumons et sacs aériens. Elle a été décrite en Afrique du Sud en $1948 \mathrm{chez}$ des canaris morts de pneumonie. Depuis lors, elle fut retrouvée dans diverses régions du globe chez le même hôte et dans les mêmes circonstances. Nous l'avons également rencontrée dans plusieurs élevages de canaris en Belgique. Sa présence était chaque fois associée à des manifestations pulmonaires graves, telles que pneumonie et inflammation purulente des sacs aériens. Cette espèce a aussi été signalée chez des perruches d'élevages.

Dans le but de déterminer l'importance des Oiseaux sauvages comme réservoir de cette espèce, nous avons entrepris, avec Hyland, de la rechercher systématiquement dans le poumon de ces hôtes. Nous avons ainsi pu démontrer qu'elle est très fréquente chez les Passériformes sauvages. Jusqu'ici, elle a été rencontrée chez 21 espèces de Passériformes appartenant à 21 genres et à 10 familles différents. C'est chez la famille Fringillidae à laquelle appartient également le canari, qu'elle est le mieux représentée. Nous l'avons également rencontrée chez Passer domesticus qui est un Ploceidae (5).

Il se confirme ainsi que ce sont bien les Oiseaux sauvages et principalement les Fringillidae qui sont les hôtes naturels de cette espèce. Le canari l'a probablement reçue de ses ancêtres sauvages et il l'a, à son tour, transmise à la perruche d'élevage. Le parasitisme du canari pourrait toutefois encore provenir d'une autre source qui nous paraît très importante, c'est celle qui est constituée par les petits Oiseaux sauvages avec lesquels les canaris entrent régulièrement en contact chez les marchands ou les éleveurs d'Oiseaux. Quant au moineau domestique, il joue probablement un rôle dans la dissémination du parasite parmi les petits Oiseaux sauvages avec lesquels il entre en contact. Peut-être que dans certaines circonstances, il pourrait aussi communiquer son parasitisme directement au canari.

\section{Résumé}

Le parasitisme par les Acariens est très répandu chez les Oiseaux et les Mammifères et il peut revêtir des formes très variées.

Une faune très nombreuse vit à la surface du corps de ces hôtes, principalement sur les plumes ou les poils, mais également sur la peau.

Il y a aussi des Acariens qui pénètrent à l'intérieur des couches superficielles de la peau ou des muqueuses. Morphologiquement, ces Acariens présentent déjà des signes évidents d'adaptation à la vie endoparasitaire, ce qui les classe plutôt parmi les endoparasites.

De toutes les formes d'acariase interne, la plus répandue est celle des voies respiratoires. Elle est observée chez les Mammifères, les Oiseaux, les Serpents, les Batraciens et même certains Mollusques.

Une autre forme d'acariase interne, plus rare, est celle du tube digestif (estomac et intestin). Elle n'a été signalée que chez les Mégachiroptères.

L'acariase des voies respiratoires est particulièrement fréquente chez les Oiseaux. Chez ces hôtes, elle est produite par des Acariens appartenant à quatre familles diffé- 
rentes. Tous ces Acariens occupent dans l'appareil respiratoire des places différentes en rapport avec leur mode d'alimentation. Deux de ces familles, les Ereynetidae et les Rhinonyssidae sont étudiées en détail en ce qui concerne l'origine du parasitisme, la spécificité et les modifications morphologiques et biologiques produites par la vie endoparasitaire.

L'étude de la distribution des espèces d'Acariens vivant dans les voies respiratoires des Oiseaux montre que c'est chez les Oiseaux aquatiques, et principalement les espèces grégaires comme les canards, que le parasitisme est le plus répandu, mais aussi le plus uniforme et le moins spécifique. Cette situation s'explique probablement par le fait que le milieu aquatique et les mœurs grégaires de beaucoup d'Oiseaux aquatiques favorisent l'échange et la dissémination de ces parasites, ce qui diminuerait les chances de spéciation et tendrait ainsi à uniformiser la faune parasitaire. L'apparition de nouvelles espèces dans un tel milieu ne peut se produire que chez les hôtes qui participent le moins activement à la vie grégaire ou encore chez les formes moins strictement aquatiques. Les faits semblent confirmer cette façon de voir. Un autre exemple de l'importance que présente l'écologie de l'hôte dans la dissémination des Acariens parasites est celui de Sternostoma tracheacolum. Cette espèce parasite le poumon de nombreux petits Passériformes sauvages. Elle est surtout fréquente chez les Fringillidae qui semblent être les hôtes naturels, mais on la rencontre aussi chez d'autres familles de Passériformes et en particulier celles avec lesquelles les Fringillidae entrent en contact. Des Fringillidae sauvages, ce parasite est passé au canari qui l'a à son tour transmis à la perruche d'élevage.

\section{Bibliographie}

FAIN A., 1957. - Les Acariens des familles Epidermoptidae et Rhinonyssidae parasites des fosses nasales d'Oiseaux au Ruanda-Urundi et au Congo Belge. Ann. Musée Congo Belge, Série 8, 60 : 1-176.

- —, 1962. - Les Acariens parasites nasicoles des Batraciens. Révision des Lawrencarinae Fain, 1957 (Ereynetidae: Trombidiformes). Bull. Inst. Roy. Sc. Nat. Belgique, XXXVIII (25) : 1-69.

- - 1963 a. - Le dimorphisme sexuel chez les Ereynetidae (Acarina: Trombidiformes). Z. f. Parasitenk., 23 : 50-62.

- —, 1963 b. - Chaetotaxie et classification des Speleognathinae. Bull. Inst. Roy. Sci. Nat. Belgique, XXXIX, 9: 1-80.

- - et HyLAND K., 1962. - The mites parasitic in the lungs of Birds. The variability of Sternostoma tracheacolum Lawrence, 1948, in domestic and wild birds. Parasitology, 52 (3-4) : 401-424.

- - et Nadchatram M., 1962. - Acariens nasicoles de Malaisie. I. Ereynetoides malayi n. g., n. sp., parasite d'un nectarin (Ereynetidae: Trombidiformes). Z. f. Parasitenk., $22: 68-82$.

Strandtmann R.W., 1958. - Host specificity of bird nasal mites (Rhinonyssidae) is a function of the Gregariousness of the host. Proc. 10 Intern. Congr. Ent., 1: 909-911.

(Institut de Médecine Tropicale, Anvers) 